\title{
Robust Adaptive Control Design for Generator Excitation
}

\author{
Zhou Lan, Deqiang Gan, Yixin Ni, Senior Member, IEEE
}

\begin{abstract}
In this paper a new nonlinear robust adaptive excitation control strategy for multi-machine power systems is presented. The designed controller is adaptive to unknown generator parameters, and robust to model errors or disturbances. It is locally implemented and independent of network topology or load conditions. In the paper the power system model is presented and the control law and adaptive law are derived. The close-loop system stability is proven. Computer test results show clearly that the proposed excitation control strategy can enhance transient stability more effectively than conventional controllers.
\end{abstract}

Index Terms-- Power system, transient stability, excitation, robust adaptive control.

\section{INTRODUCTION}

$\mathrm{I}^{\mathrm{n}}$ recent years, there has been an increasing interest in applying various advanced nonlinear control technologies to power systems. The most successful ones may be the applications of differential geometric theory and robust control theories. With the application of differential geometric theory, nonlinear power systems can be transformed into linear ones, and then various conventional linear control theories can be used to provide good performance ${ }^{[1-3]}$. It must be noted that nonlinear control design based on differential geometric approach is much better than conventional linear design based on linearization of the system at one or more operating points, but it is generally based on the exact knowledge of mathematic model of the power system, without considering uncertainties like model errors, parameter errors or any kind of disturbances. In order to overcome this limitation, various robust control theories have been suggested to enhance the control robustness of power systems ${ }^{[4-7]}$. Based on the linear model of a power system, linear $H_{\infty}$ robust control theory can be applied to minimize the impact of disturbances on the output of the system by solving Riccati equations to obtain the 'optimal' control law against the 'worst' disturbance ${ }^{[4]}$, while Lyapunov theory can be applied directly to guarantee the stability of the close-loop system ${ }^{[5]}$, and newly developed LMI (Linear Matrix Inequality)

This work was supported by the National Key Basic Research Special Fund (Project 2004CB217900) of China and the National Natural Science Foundation (Project 50337010) of China.

Zhou Lan and Deqiang Gan are with the Dept. of EE, Zhejiang Univ., Hongzhou, China (e-mail: lanzhou_zju@163.com; dgan@zju.edu.cn).

Yixin Ni is with the Dept. of EEE, the Univ. of Hong Kong, Hong Kong, China. (e-mail: yxni@eee.hku.hk). approach can be also applied to obtain the 'optimal' linear control law by solving an optimization problem constrained by matrix inequalities ${ }^{[6]}$. Based on the nonlinear model of a power system, a recursive design method, which avoids solving HJI (Hamilton Jacobi Issacs) inequalities, was proposed to solve the nonlinear $H_{\infty}$ robust control problems in power systems ${ }^{[7]}$.

There are many parameters either totally unknown or slowly time-varying in a power system. For example, the damping coefficient of a generator is usually untraceable; the synchronous reactance and transient reactance may vary slowly during system dynamics because of saturation effect; and the parameters of transmission lines may be unknown or change slowly with the environmental condition. In those cases, adaptive control, using dynamic estimation of unknown parameters, is more appropriate and attractive to solve the above unknown-parameter problems ${ }^{[8-10]}$. The adaptive control theories for linear systems are relatively mature, while for nonlinear systems, the nonlinear adaptive control has no universal approaches and is still under development.

Recently, robust adaptive control (RAC) of nonlinear systems has been widely studied and fruitful results have been obtained ${ }^{[11-15]}$. On the other hand, power system RAC has become a research hotspot in recent years. The objective of this paper is to apply the back-stepping method proposed in [11] to multi-machine power system excitation control.

The suggested controller is locally implemented, so does not need system information communication, and is also independent of network topology and load conditions. The designed controller is adaptive to uncertain or unknown generator damping coefficient, d-axis synchronous reactance and transient reactance, and is robust to model errors or disturbances. It is proved that the suggested novel controller can guarantee uniformly ultimately boundedness of all system states and drive the machine angle of each generator to a small neighborhood of the given ideal value. A 4-machine interconnected power system is used for computer test. Computer test results show clearly the effectiveness and the special feature of the controller.

\section{The Dynamic Model Of A Multi Machine Power SYSTEM}

Assume there is a multi-machine power system consisting of $N$ generators. As is well known, the $\mathrm{i}^{\text {th }}$ generator, with proper assumptions and with bounded model errors or 
disturbances represented by $\Delta_{i 1}$ and $\Delta_{i 2}$, can be represented by a third order model below ${ }^{[4]}$

$$
\left\{\begin{array}{l}
\dot{\delta}_{i}=\omega_{i}-\omega_{0} \\
\dot{\omega}_{i}=\frac{\omega_{0}}{M_{i}}\left(P_{m i}-P_{e i}\right)-\frac{D_{i}}{M_{i}}\left(\omega_{i}-\omega_{0}\right)+\Delta_{i 1} \\
\dot{E}_{q i}^{\prime}=\frac{1}{T_{d 0 i}^{\prime}}\left(E_{f i}-E_{q i}^{\prime}-\left(X_{d i}-X_{d i}^{\prime}\right) I_{d i}\right)+\Delta_{i 2}
\end{array}\right.
$$

where $P_{e i}=E_{q i}^{\prime} I_{q i}$.

The notation for the model is given in [3]. Supposing that $D_{i}, X_{d i}$ and $X_{d i}^{\prime}$ are unknown bounded parameters, the designed controller should be adaptive to these uncertain parameters and be robust to model errors or disturbances represented by $\Delta_{i 1}$ and $\Delta_{i 2}$.

Now, we define the coordinate transformation as

$$
\mathbf{x}_{i}=\left[\delta_{i}-\delta_{i}^{d}, \omega_{i}-\omega_{0}, \frac{\omega_{0}}{M_{i}}\left(P_{m i}-P_{e i}\right)\right]^{T}
$$

where $\delta_{i}^{d}$ is the given ideal operation point of machine power angle. And we define the output of the subsystem as $y_{i}(t)=\delta_{i}-\delta_{i}^{d}$. The control object is to drive the output $y_{i}$ to a small neighborhood of the origin, which is equivalent to driving the machine angle $\delta_{i}$ to a small neighborhood of the given idea value $\delta_{i 0}$.

In the new coordinate, (1) can be transformed into the following perturbed exact-feedback nonlinear form ${ }^{\text {[11-15] }}$

$$
\left\{\begin{array}{l}
\dot{x}_{i 1}=x_{i 2} \\
\dot{x}_{i 2}=x_{i 3}+\boldsymbol{\theta}_{i}^{T} \boldsymbol{\varphi}_{i 1}+d_{i 1} \\
\dot{x}_{i 3}=v_{i}+\boldsymbol{\theta}_{i}^{T} \boldsymbol{\varphi}_{i 2}+d_{i 2} \\
y_{i}=x_{i 1}
\end{array}\right.
$$

where $v_{i}=\frac{\omega_{0}}{M_{i}}\left(-\frac{I_{q i}}{T_{d 0 i}^{\prime}} E_{f i}+\frac{P_{e i}}{T_{d 0 i}^{\prime}}-E_{q i}^{\prime} \dot{I}_{q i}\right)$ is the virtual control input and

$$
\begin{aligned}
& \boldsymbol{\theta}_{i}=\left[\theta_{i 1}, \theta_{i 2}\right]^{T}, \boldsymbol{\varphi}_{i 1}=\left[\varphi_{i 11}, \varphi_{i 12}\right]^{T}, \boldsymbol{\varphi}_{i 2}=\left[\varphi_{i 21}, \varphi_{i 22}\right]^{T} ; \\
& \theta_{i 1}=D_{i}, \theta_{i 2}=X_{d i}-X_{d i}^{\prime} ; \\
& \varphi_{i 11}=-\frac{x_{i 2}}{M_{i}}, \varphi_{i 22}=\frac{\omega_{0} I_{d i} I_{q i}}{M_{i} T_{d 0 i}^{\prime}}, \varphi_{i 12}=\varphi_{i 21}=0 ; \\
& d_{i 1}=\Delta_{i 1}, d_{i 2}=-\frac{\omega_{0} I_{q i}}{M_{i}} \Delta_{i 2} .
\end{aligned}
$$

We can easily know that $d_{i 1}$ and $d_{i 2}$ are all bounded. Obviously, the equilibrium point of (2) is the origin.

\section{Robust AdAPtive Controller Design}

Now we shall show a back-stepping design procedure of the nonlinear robust adaptive controller for the system defined in (2).

The control design is based on the claim below

Claim. The following inequality holds for any $\varepsilon>0$ and for any $u \in R$

$$
0 \leq|u|-u \tanh \left(\frac{u}{\varepsilon}\right) \leq \delta \varepsilon
$$

where $\delta$ is a constant that satisfies $\delta=e^{-(\delta+1)}$, i.e. $\delta=0.2785$.

Firstly, we consider the transformation below

$$
\left\{\begin{array}{l}
z_{i 1}=x_{i 1} \\
z_{i 2}=x_{i 2}+k_{i 1} x_{i 1} \\
z_{i 3}=x_{i 3}-\alpha_{i}
\end{array}\right.
$$

where $k_{i 1}$ is a given positive constant; $\alpha_{i}$ is a smooth function which satisfies $\left.\alpha_{i}\right|_{\mathbf{x}_{i}=0}=0$. It can be seen that the equilibrium point of the new subsystem (3) is still the origin.

Because $d_{i 1}$ is bounded, we have $\left|d_{i 1}\right| \leq \psi_{i 1}$, where $\psi_{i 1}$ is an unknown positive constant.

Consider the Lyapunov function

$$
V_{i}^{\prime}=\frac{1}{2} z_{i 1}^{2}+\frac{1}{2} z_{i 2}^{2}+\frac{1}{2} \tilde{\boldsymbol{\theta}}_{i}^{T} \boldsymbol{\Gamma}_{i}^{-1} \tilde{\boldsymbol{\theta}}_{i}+\frac{1}{2 \gamma_{i 1}} \tilde{\psi}_{i 1}^{2}
$$

where $\gamma_{i 1}$ is a given positive constant; $\tilde{\psi}_{i 1}=\widehat{\psi}_{i 1}-\psi_{i 1}^{M}$ is parameter estimation error; $\widehat{\psi}_{i 1}$ is the dynamic estimation of $\psi_{i 1}^{M}$, and $\psi_{i 1}^{M}=\max \left\{\psi_{i 1}, \psi_{i 1}^{0}\right\} ; \psi_{i 1}^{0}$ is a given positive constant; $\tilde{\boldsymbol{\theta}}_{i}=\widehat{\boldsymbol{\theta}}_{i}-\boldsymbol{\theta}_{i}$ is a vector of parameter estimation errors; $\hat{\boldsymbol{\theta}}_{i}$ is the dynamic estimation of $\boldsymbol{\theta}_{i} ; \boldsymbol{\Gamma}_{i}$ is a given positive constant matrix.

The time derivative is given by

$$
\begin{aligned}
\dot{V}_{i}^{\prime}= & z_{i 2}\left(k_{i 1} x_{i 2}+z_{i 1}+z_{i 3}+\alpha_{i}+\boldsymbol{\theta}_{i}^{T} \boldsymbol{\varphi}_{i 1}+d_{i 1}\right) \\
& -k_{i 1} z_{i 1}^{2}+\tilde{\boldsymbol{\theta}}_{i}^{T} \boldsymbol{\Gamma}_{i}^{-1} \dot{\widehat{\boldsymbol{\theta}}}_{i}+\frac{1}{\gamma_{i 1}} \tilde{\psi}_{i 1} \dot{\vec{\psi}}_{i 1}
\end{aligned}
$$

Defining $\alpha_{i}=-z_{i 1}-k_{i 1} x_{i 2}-k_{i 2} z_{i 2}-\beta_{i 1}-\widehat{\boldsymbol{\theta}}_{i}^{T} \boldsymbol{\varphi}_{i 1}$, where $k_{i 2}$ is a given positive constant and $\beta_{i 1}$ is the compensation for $d_{i 1}$, we have

$$
\begin{aligned}
\dot{V}_{i}^{\prime}= & -k_{i 1} z_{i 1}^{2}-k_{i 2} z_{i 2}^{2}+z_{i 2} z_{i 3}+\tilde{\boldsymbol{\theta}}_{i}^{T}\left(\boldsymbol{\Gamma}_{i}^{-1} \dot{\overrightarrow{\boldsymbol{\theta}}}_{i}-z_{i 2} \boldsymbol{\varphi}_{i 1}\right) \\
& +z_{i 2}\left(d_{i 1}-\beta_{i 1}\right)+\frac{1}{\gamma_{i 1}} \tilde{\psi}_{i 1} \dot{\bar{\psi}}_{i 1}
\end{aligned}
$$

Now, we define

$$
\left\{\begin{array}{l}
\dot{\vec{\psi}}_{i 1}=\gamma_{i 1}\left[z_{i 2} w_{i 1}-\sigma_{i 1}\left(\widehat{\psi}_{i 1}-\psi_{i 1}^{0}\right)\right] \\
\beta_{i 1}=\widehat{\psi}_{i 1} w_{i 1} \\
w_{i 1}=\tanh \left(\frac{z_{i 2}}{\varepsilon_{i 1}}\right)
\end{array}\right.
$$

where $\sigma_{i 1}$ and $\varepsilon_{i 1}$ are given positive constants.

From the claim and (6), we can easily know

$$
z_{i 2}\left(d_{i 1}-\beta_{i 1}\right)+\frac{1}{\gamma_{i 1}} \tilde{\psi}_{i 1} \dot{\bar{\psi}}_{i 1} \leq \frac{\varepsilon_{i 1}}{2} \psi_{i 1}^{M}-\sigma_{i 1} \tilde{\psi}_{i 1}\left(\widehat{\psi}_{i 1}-\psi_{i 1}^{0}\right)
$$

Because $-\sigma_{i 1} \tilde{\psi}_{i 1}\left(\widehat{\psi}_{i 1}-\psi_{i 1}^{0}\right) \leq-\frac{\sigma_{i 1}}{2} \tilde{\psi}_{i 1}^{2}+\frac{\sigma_{i 1}}{2}\left(\psi_{i 1}^{M}-\psi_{i 1}^{0}\right)^{2}$, we arrive at the following result

$$
z_{i 2}\left(d_{i 1}-\beta_{i 1}\right)+\frac{1}{\gamma_{i 1}} \tilde{\psi}_{i 1} \dot{\vec{\psi}}_{i 1} \leq-\frac{\sigma_{i 1}}{2} \tilde{\psi}_{i 1}^{2}+\lambda_{i 1}
$$


where $\lambda_{i 1}=\frac{\varepsilon_{i 1}}{2} \psi_{i 1}^{M}+\frac{\sigma_{i 1}}{2}\left(\psi_{i 1}^{M}-\psi_{i 1}^{0}\right)^{2}$.

Substituting (17) into (15), we have

$$
\begin{aligned}
\dot{V}_{i}^{\prime} \leq & -k_{i 1} z_{i 1}^{2}-k_{i 2} z_{i 2}^{2}-\frac{\sigma_{i 1}}{2} \tilde{\psi}_{i 1}^{2}+\lambda_{i 1} \\
& +z_{i 2} z_{i 3}+\tilde{\boldsymbol{\theta}}_{i}^{T} \boldsymbol{\Gamma}_{i}^{-1}\left(\dot{\boldsymbol{\theta}}_{i}-z_{i 2} \boldsymbol{\Gamma}_{i} \boldsymbol{\varphi}_{i 1}\right)
\end{aligned}
$$

The time derivative of $\alpha_{i}$ can be expressed as

$$
\dot{\alpha}_{i}=d \alpha_{f i}-\frac{\partial \alpha_{i}}{\partial x_{i 2}} \tilde{\boldsymbol{\theta}}_{i}^{T} \boldsymbol{\varphi}_{i 1}+\frac{\partial \alpha_{i}}{\partial x_{i 2}} d_{i 1}
$$

where $d \alpha_{f i}=\frac{\partial \alpha_{i}}{\partial x_{i 1}} x_{i 2}+\frac{\partial \alpha_{i}}{\partial \widehat{\psi}_{i 1}} \dot{\bar{\psi}}_{i 1}+\frac{\partial \alpha_{i}}{\partial \widehat{\boldsymbol{\theta}}_{i}} \dot{\boldsymbol{\theta}}_{i}+\frac{\partial \alpha_{i}}{\partial x_{i 2}}\left(x_{i 3}+\widehat{\boldsymbol{\theta}}_{i}^{T} \boldsymbol{\varphi}_{i 1}\right)$, and $\frac{\partial \alpha_{i}}{\partial x_{i 2}}=-\left(k_{i 1}+k_{i 2}\right)-\widehat{\psi}_{i 1}\left(1-w_{i 1}^{2}\right) / \varepsilon_{i 1}+\widehat{\theta}_{i 1} / M_{i}$.

Because $d_{i 1}$ and $d_{i 2}$ are all bounded and $\frac{\partial \alpha_{i}}{\partial x_{i 2}}$ is bounded too, we know $d_{i 3}=d_{i 2}-\frac{\partial \alpha_{i}}{\partial x_{i 2}} d_{i 1}$ is also bounded. So we define $\left|d_{i 3}\right| \leq \psi_{i 2}$, where $\psi_{i 2}$ is an unknown positive constant.

Now, we define the Lyapunov function of the whole subsystem as

$$
V_{i}=V_{i}^{\prime}+\frac{1}{2} z_{i 3}^{2}+\frac{1}{2 \gamma_{i 2}} \tilde{\psi}_{i 2}^{2}
$$

where $\gamma_{i 2}$ is a given positive constant; $\tilde{\psi}_{i 2}=\widehat{\psi}_{i 2}-\psi_{i 2}^{M}$ is parameter estimation error; $\widehat{\psi}_{i 2}$ is the dynamic estimation of $\psi_{i 2}^{M}$, and $\psi_{i 2}^{M}=\max \left\{\psi_{i 2}, \psi_{i 2}^{0}\right\} ; \psi_{i 2}^{0}$ is a given positive constant.

The time derivative of (9) is given by

$$
\begin{aligned}
\dot{V}_{i} \leq & -k_{i 1} z_{i 1}^{2}-k_{i 2} z_{i 2}^{2}-\frac{\sigma_{i 1}}{2} \tilde{\psi}_{i 1}^{2}+\lambda_{i 1} \\
& +z_{i 3}\left(v_{i}+z_{i 2}+\boldsymbol{\theta}_{i}^{T} \boldsymbol{\varphi}_{i 2}+d_{i 2}-\dot{\alpha}_{i}\right) \\
& +\tilde{\boldsymbol{\theta}}_{i}^{T} \boldsymbol{\Gamma}_{i}^{-1}\left(\dot{\widehat{\boldsymbol{\theta}}}_{i}-z_{i 2} \boldsymbol{\Gamma}_{i} \boldsymbol{\varphi}_{i 1}\right)+\frac{1}{\gamma_{i 2}} \tilde{\psi}_{i 2} \dot{\bar{\psi}}_{i 2}
\end{aligned}
$$

Now defining the virtual control law as

$$
v_{i}=-z_{i 2}-k_{i 3} z_{i 3}-\widehat{\boldsymbol{\theta}}_{i}^{T} \boldsymbol{\varphi}_{i 2}-\beta_{i 2}+d \alpha_{f i}
$$

where $k_{i 3}$ is a given positive constant and $\beta_{i 2}$ is the compensation term for $d_{i 3}$, we can have

$$
\begin{aligned}
\dot{V}_{i} \leq & -k_{i 1} z_{i 1}^{2}-k_{i 2} z_{i 2}^{2}-k_{i 3} z_{i 3}^{2}-\frac{\sigma_{i 1}}{2} \tilde{\psi}_{i 1}^{2}+\lambda_{i 1} \\
& +z_{i 3}\left(d_{i 3}-\beta_{i 2}\right)+\frac{1}{\gamma_{i 2}} \tilde{\psi}_{i 2} \dot{\bar{\psi}}_{i 2} \\
& \left.+\tilde{\boldsymbol{\theta}}_{i}^{T}\left[\boldsymbol{\Gamma}_{i}^{-1} \dot{\widehat{\boldsymbol{\theta}}}_{i}-\left(z_{i 2}-z_{i 3} \frac{\partial \alpha_{i}}{\partial x_{i 2}}\right) \boldsymbol{\varphi}_{i 1}-z_{i 3} \boldsymbol{\varphi}_{i 2}\right)\right]
\end{aligned}
$$

It is similar to (7) that if we define

$$
\left\{\begin{array}{l}
\dot{\bar{\psi}}_{i 2}=\gamma_{i 2}\left[z_{i 3} w_{i 2}-\sigma_{i 2}\left(\widehat{\psi}_{i 2}-\psi_{i 2}^{0}\right)\right] \\
\beta_{i 2}=\widehat{\psi}_{i 2} w_{i 2} \\
w_{i 2}=\tanh \left(\frac{z_{i 3}}{\varepsilon_{i 2}}\right)
\end{array}\right.
$$

where $\sigma_{i 2}$ and $\varepsilon_{i 2}$ are given positive constants, we can have

$$
\begin{aligned}
& z_{i 3}\left(d_{i 2}-\frac{\partial \alpha_{i}}{\partial x_{i 2}} d_{i 1}-\beta_{i 2}\right)+\frac{1}{\gamma_{i 2}} \tilde{\psi}_{i 2} \dot{\hat{\psi}}_{i 2} \\
& \leq-\frac{\sigma_{i 2}}{2} \tilde{\psi}_{i 2}^{2}+\lambda_{i 21}
\end{aligned}
$$

where $\lambda_{i 21}=\frac{\varepsilon_{i 2}}{2} \psi_{i 2}^{M}+\frac{\sigma_{i 2}}{2}\left(\psi_{i 2}^{M}-\psi_{i 2}^{0}\right)^{2}$.

Now, the adaptive law of $\widehat{\boldsymbol{\theta}}_{i}$ is given as

$$
\dot{\hat{\boldsymbol{\theta}}}_{i}=\boldsymbol{\Gamma}_{i}\left[\left(z_{i 2}-\frac{\partial \alpha_{i}}{\partial x_{i 2}} z_{i 3}\right) \boldsymbol{\varphi}_{i 1}+z_{i 3} \boldsymbol{\varphi}_{i 2}-\sigma_{\theta i}\left(\tilde{\boldsymbol{\theta}}_{i}-\boldsymbol{\theta}_{i}^{0}\right)\right]
$$

where $\sigma_{\theta i}$ is a given positive constant and $\boldsymbol{\theta}_{i}^{0}$ is a given constant vector.

Using the adaptive law of (14), we know that

$$
\begin{aligned}
& \left.\tilde{\boldsymbol{\theta}}_{i}^{T}\left[\boldsymbol{\Gamma}_{i}^{-1} \dot{\overrightarrow{\boldsymbol{\theta}}}_{i}-\left(z_{i 2}-z_{i 3} \frac{\partial \alpha_{i}}{\partial x_{i 2}}\right) \boldsymbol{\varphi}_{i 1}-z_{i 3} \boldsymbol{\varphi}_{i 2}\right)\right] \\
& =-\sigma_{\theta i} \tilde{\boldsymbol{\theta}}_{i}^{T}\left(\tilde{\boldsymbol{\theta}}_{i}-\boldsymbol{\theta}_{i}^{0}\right) \leq-\frac{\sigma_{\theta i}}{2} \tilde{\boldsymbol{\theta}}_{i}^{T} \tilde{\boldsymbol{\theta}}_{i}+\lambda_{i 22}
\end{aligned}
$$

where $\lambda_{i 22}=\frac{\sigma_{\theta i}}{2}\left|\boldsymbol{\theta}_{i}-\boldsymbol{\theta}_{i}^{0}\right|^{2}$.

Substituting (13) and (15) into (11), we have

$$
\begin{aligned}
\dot{V}_{i} \leq & -k_{i 1} z_{i 1}^{2}-k_{i 2} z_{i 2}^{2}-k_{i 3} z_{i 3}^{2}-\frac{\sigma_{i 1}}{2} \tilde{\psi}_{i 1}^{2}-\frac{\sigma_{i 2}}{2} \tilde{\psi}_{i 2}^{2}-\frac{\sigma_{\theta i}}{2} \tilde{\boldsymbol{\theta}}_{i}^{T} \tilde{\boldsymbol{\theta}}_{i} \\
& +\lambda_{i}
\end{aligned}
$$

where $\lambda_{i}=\lambda_{i 1}+\lambda_{i 21}+\lambda_{i 22}$.

$$
\text { Let } c_{i}=\min \left\{2 k_{i 1}, 2 k_{i 2}, 2 k_{i 3}, \sigma_{i 1} \gamma_{i 1}, \sigma_{i 2} \gamma_{i 2}, \frac{\sigma_{\theta i}}{\lambda_{\min }\left(\boldsymbol{\Gamma}_{i}^{-1}\right)}\right\} \text {, we }
$$
have

$$
\dot{V}_{i} \leq-c_{i} V_{i}+\lambda_{i}
$$

The above equation guarantees that

(a) $\mathbf{z}_{i}, \tilde{\boldsymbol{\theta}}_{i}, \tilde{\psi}_{i 1}, \tilde{\psi}_{i 2}$ are all uniformly ultimately bounded;

(b) Given any constant $\mu_{i}>\mu_{i}^{*}=\sqrt{2 \lambda_{i} / c_{i}}$, there exists $T>0$ such that, for $T \leq t \leq \infty,\left|z_{i j}(t)\right| \leq \mu_{i},(j=1,2,3)$.

Because $y_{i}=x_{i 1}=z_{i 1}$, the output $y_{i}$ is regulated to a small neighborhood of zero, as shown in (b).

Finally, the excitation control law for the $i^{\text {th }}$ generator can be derived by

$$
E_{f i}=\frac{T_{d 0 i}^{\prime}}{I_{q i}}\left(-\frac{M_{i}}{\omega_{0}} v_{i}+\frac{P_{e i}}{T_{d 0 i}^{\prime}}-E_{q i}^{\prime} \dot{I}_{q i}\right)
$$

The control law in (17) is always valid since in general $I_{q i} \neq 0$. Under this control law, the machine angle $\delta_{i}$ will be driven to a small neighborhood of the given ideal value $\delta_{i}^{d}$. 
Remark1. The model (2) is based on local dynamic information, so the controller designed will be local and decentralized, and independent of network topology or load conditions.

Remark2. It is clear that the designed controller is adaptive to uncertain parameters $D_{i}, X_{d i}$ and $X_{d i}^{\prime}$. In fact, the changes of uncertain parameters within limited bounds in the dynamic are very small disturbances to the system, and the controller can stabilize the system rapidly so is said to be adaptive to parameter uncertainties.

Remark3. The designed controller is robust to bounded model errors or disturbances. In the control design, the bounds of errors are not fixed and are estimated by dynamic estimators. The advantage of the estimator approach is that it can improve the system performance noticeably if we set $\psi_{i 1}^{0}$ and $\psi_{i 2}^{0}$ to be very small since the controller allows large error during transients, while it may be very small when the system settles in steady state.

Remark4. Because $\dot{I}_{q i}$ can not be obtained from its equation, we can use a tracking differentiator (TD), which can track the differential of the input signal with high precision and fast converging speed ${ }^{[16]}$, to obtain the tracking signal of $\dot{I}_{q i}$. In (1), we assume $P_{m i}$ as constant. Actually there is no difficulty to consider different governor dynamic models if $P_{m i}$ dynamics can be obtained from a TD.

\section{COMPuter Simulation Studies}

The computer test has been conducted on a 4-machine interconnected power system ${ }^{[17]}$ (see Fig. 1). Time simulation is conducted to verify the effectiveness of the suggested new controller. The system data can be found in [18].

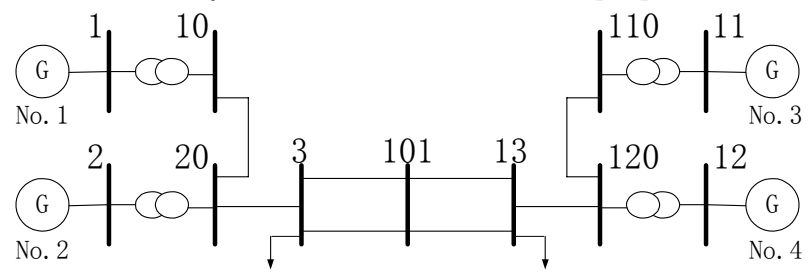

Fig. 1. A 4-machine test power system single-line diagram

In the time simulation, all the machines use the $6^{\text {th }}$ order model with $\left(\delta_{i}, \omega_{i}, E_{q i}^{\prime}, E_{d i}^{\prime}, E_{q i}^{\prime \prime}, E_{d i}^{\prime \prime}\right)$ as state variables and saturation effect of all machines is considered. The governor dynamics are neglected. The loads are represented by constant impedances. The output limits of all exciters are set as $[0,5]$.

Desired uncertain parameter values, which are different from real values, are given to be the initial values of the estimation dynamic. They are:

$$
\boldsymbol{\theta}_{i}^{0}=[3,1]^{T}, \psi_{i 1}^{0}=\psi_{i 2}^{0}=0 . \quad(i=1 \sim 4)
$$

The parameters of AVR+PSS controllers are optimized so that the controllers have good enough performances, while the control parameters are carefully selected as below:

$$
\begin{aligned}
& k_{i 1}=1, k_{i 2}=3, k_{i 3}=5, \gamma_{i 1}=\gamma_{i 2}=10 \\
& \varepsilon_{i 1}=\varepsilon_{i 2}=\sigma_{i 1}=\sigma_{i 2}=\sigma_{\theta i}=1 \\
& \boldsymbol{\Gamma}_{i}=\left[\begin{array}{cc}
10 & 0 \\
0 & 10
\end{array}\right] \quad(i=1 \sim 4)
\end{aligned}
$$

The fault considered in the simulation is: a 3-phase short fault happens on line 101-3 close to bus 101 at $0.1 \mathrm{~s}$; the fault line trips at $0.2 \mathrm{~s}$; line $13-101$ is tripped for an unknown reason at $\mathrm{t}=4 \mathrm{~s}$.

The effect of the designed nonlinear robust adaptive controller (NRAC) and the conventional AVR+PSS excitation system are compared through rotor angle dynamics with generator 4 taken as reference machine and four machines all equipped with NRAC and AVR+PSS respectively.

Machine angle swings in the test case are shown in Fig. 2.

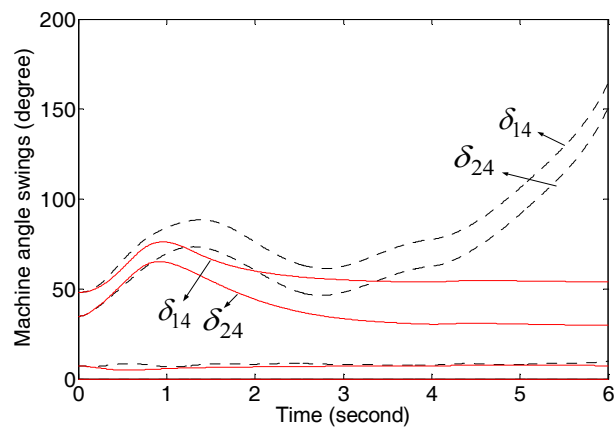

Fig. 2. Machine angle swing dynamics (--- AVR+PSS; - NRAC)

From Fig. 2 we can see that the designed new controllers behave very well in improving power system transient stability when the system is subject to large disturbances. The new controllers not only improve first-swing stability of machine angles but also damp angle swings more quickly than conventional exciters, as we can see from Fig. 2.

In the test case, the machine angles are all driven to the desired values which are given to be equal to the initial values in the simulation, so the angle swings all converge to the neighborhood of their initial values, which can be seen from the figure above.

Parameter estimation dynamics are shown in Fig. 3.

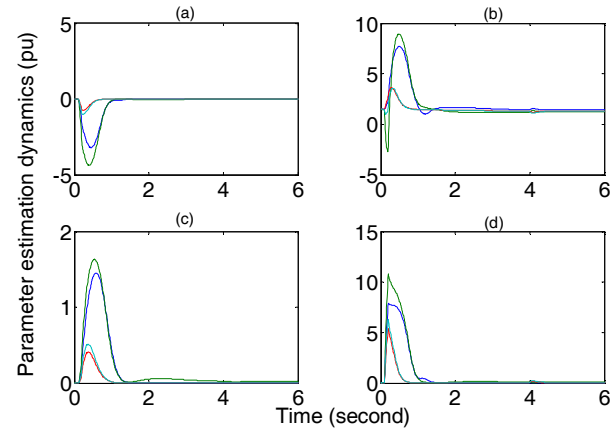

Fig. 3. Parameter estimation dynamics ((a)-(d) represents $\widehat{\theta}_{i 1}, \widehat{\theta}_{i 2}, \widehat{\psi}_{i 1}$ and $\widehat{\psi}_{i 2}$ respectively)

It can be seen that the parameter estimations all converged to the neighborhoods of the given initial values very quickly. In fact, we don't care too much about how much the exact parameters are; but we do care if the estimated parameters can converge quickly to an acceptable neighborhood of the given 
ideal values. Therefore the plots in Fig. 3 show the adaptive controller has acceptable dynamic performance.

Machine terminal voltages are shown in Fig. 4. The terminal voltages are expected to be within 0.9 and 1.1. From Fig. 4 we can see that machine terminal voltages are all acceptable.

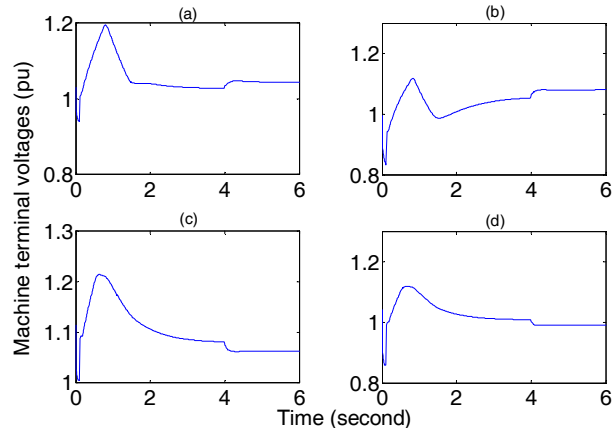

Fig. 4 Machine terminal voltages ((a)-(d) represents terminal voltage of machine 1-4 respectively)

Machine excitation voltages (controller output) are shown in Fig. 5. The output limits are set as $[0,5]$ pu. It can be seen in the figure that the controllers agilely stabilized the power system angle swing through its fast regulation after faults happened. The curves are smooth and bounded, so is realizable.

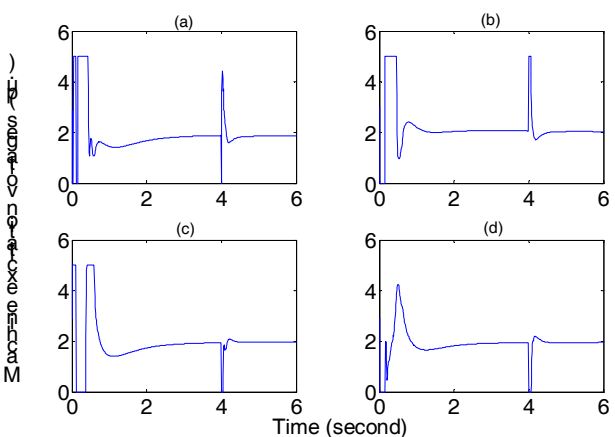

Fig. 4 Machine excitation voltages ((a)-(d) represents excitation voltage of machine $1-4$ respectively)

\section{CONCLUSION}

A nonlinear robust adaptive excitation control has been proposed for multi-machine power systems. The designed controller is locally implemented and de-coupled with the network and system loading conditions. The designed controller is robust to model errors or disturbances and adaptive to unknown machine parameters. It is proved that the controller design guarantees the uniform ultimate boundedness of all system states; and the power angles can converge to the neighborhoods of the given ideal values. Computer test results demonstrate the effectiveness of the suggested controller. The problems of optimal selection of controller parameters and optimal allocation of the controllers in a power system are worth further study.

\section{REFERENCES}

[1] Y. Wang, D. J. Hill and L. Gao, "Transient stability enhancement and voltage regulation of power systems," IEEE Trans. Power Systems, vol. 8, no. 2, pp. 549-557, 1993.
[2] J. W. Chapman, M. D. Ilic and C. A. King, "Stabilizing a multimachine power system via decentralized feedback linearizing excitation control," IEEE Trans. Power Systems, vol. 8, no. 3, pp. 830-839, 1993.

[3] Q. Lu, Y. Sun and Z. Xu, "Decentralized nonlinear optimal excitation control," IEEE Trans. Power Systems, vol. 11, no. 4, pp. 1957-1962, 1996.

[4] C. Sun, Z. Zhao and Y. Sun, "Design of nonlinear robust excitation control for multimachine power systems," IEE Proc. - Gener. Transm. Distrib., vol. 143, no. 3, pp. 253-257, 1996.

[5] D. Gan, Z.Qu and H. Cai, "Multi machine power system excitation control design via theories of feedback linearization control and nonlinear robust control," International Journal of Systems Science, vol. 31, no. 4, pp. 519-527, 2000.

[6] Aleksandar I. Zecevic, Gordana Neskovic and Dragoslav D. Siljak, "Robust decentralized exciter control with linear feedback," IEEE Trans. Power Systems, vol. 19, no. 2, pp. 1096-1103, 2004.

[7] Q. Lu, S. Mei and W. Hul, "Nonlinear decentralized disturbance attenuation excitation control via new recursive design for multimachine power systems," IEEE Trans. Power Systems, vol. 16, no. 4, pp. 729-736, 2001.

[8] H. Jiang, J. F. Dorsey and Z. Qu, "Global robust adaptive control of power systems," IEE Proc.- Gener. Transm. Distrib., vol. 141, no. 5, pp. 429-436, 1994.

[9] S. Jain, F. Khorrami and B. Fardanesh, "Adaptive Nonlinear Excitaiton Control of Power Systems with Unknown Interconnections," IEEE Trans. Control Systems Technology, vol. 2, no. 4, pp. 436-445, 1994.

[10] T. Shen, S. Mei and Q. Lu, "Adaptive nonlinear excitation control with $L_{2}$ disturbance attenuation for power systems," Automatica, vol. 39, pp. 81-89, 2003.

[11] M. M. Polycarpou and P. A. Ioannou, "A robust adaptive nonlinear control design," Automatica, vol. 32, no. 3, pp. 423-427, 1996.

[12] Z. Jiang and D. J. Hill, "A robust adaptive backstepping scheme for nonlinear systems with unmodeled dynamics," IEEE Trans. Automatic Control, vol. 44, no. 9, pp. 1705-1711, 1999.

[13] R. Marino and P. Tomei. "Robust adaptive state-feedback tracking for nonlinear systems," IEEE Trans. Automatic Control, vol. 43, no. 1, pp. 84-89, 1998.

[14] S. S. Ge and J. Wang, "Robust adaptive tracking for time-varying uncertain nonlinear systems with unknown control coefficients," IEEE Trans. Automatic Control, vol. 48, no. 8, pp. 1463-1469, 2003.

[15] Y. Liu and X. Y. Li, "Robust adaptive control of nonlinear systems with unmodelled dynamics," IEE Proc.-Control Theory Appl., vol. 151, no. 1, pp. 83-88, 2004.

[16] Xuejun Wang, Stephen Yau and Jie Huang, "A study of trackingdifferentiator," in Proc. 2000 IEEE Power Engineering Society Decision and Control Conf., pp. 4783-4784.

[17] P. Kundur, Power System Stability and Control, McGraw-Hill, 1994.

\section{BIOGRAPHIES}

Zhou Lan is a Ph.D. candidate in Zhejiang University. His research interest is in power system stability and control.

Deqiang Gan received the Ph.D. degree in electrical engineering from Xian Jiaotong University, Xian, China, in 1994. Currently, he is with Zhejiang University, Zhejiang Province, China. He was a Senior Analyst in ISO New England, Inc., Holyoke, MA, where he worked on issues related to the design, implementation, and economic analysis of electricity markets. Prior to joining ISO New England, Inc., he held research positions at several universities in the U.S. and Japan.

Yixin Ni (S. M.'92) received her B. Eng., M. Eng. and Ph. D. degrees all from Tsinghua University, China. She was a former professor and director of National Power System Lab, Tsinghua Univ. and is currently with the Univ. of Hong Kong. Her research interests are in power system stability and control, HVDC transmission, FACTS, and power markets. 